\title{
ON HEAT CONDUCTION IN MATERIALS WITH MEMORY*
}

\author{
BY \\ JACE W. NUNZIATO \\ Sandia Laboratories, Albuquerque, New Mexico
}

1. Introduction. There exists considerable experimental evidence that heat transfer by conduction in many materials can be accurately described by the classical linear theory of heat conduction. This theory, for homogeneous and isotropic rigid bodies, is based on Fourier's law

$$
q=-\kappa \nabla \theta
$$

in which the heat flux $q$ is a linear function of the temperature gradient $\nabla \theta$ and the thermal conductivity $\kappa$ is a positive constant. It is further assumed that the internal energy $e$ depends linearly on the temperature difference $\theta-\theta_{0}$ :

$$
e=e_{0}+\alpha\left(\theta-\theta_{0}\right)
$$

where the heat capacity $\alpha$ is also a positive constant. Then Eqs. (1.1) and (1.2) combine with the energy balance equation to yield the classical linear heat equation

$$
\dot{\theta}=(\kappa / \alpha) \nabla^{2} \theta .
$$

This theory has, however, two principal shortcomings. First, it is unable to account for memory effects which may be prevalent in some materials, particularly at low temperatures. Secondly, the parabolic heat equation (1.3) predicts an unrealistic result: that a thermal disturbance at one point of the body is instantly felt everywhere in the body (though not equally). This implies that in Fourier heat conductors, finite thermal discontinuities must propagate with infinite speed. It is these observations which lead one to believe that for materials with memory, Fourier's law (1.1) may be a limiting approximation (perhaps for sufficiently steady temperature fields) to a more general nonlinear constitutive assumption relating the heat flux $\boldsymbol{q}$ to the material's thermal history.

Gurtin and Pipkin [17] have proposed one such nonlinear memory theory of heat conduction which is independent of the present value of the temperature gradient. This theory generalizes constitutive relations deduced from kinetic theory by Maxwell [19] and Cattaneo [2] and has associated with it finite wave speeds. ${ }^{1}$ Moreover, when this theory is linearized, it yields the heat flux relation

$$
\mathbf{q}(t)=-\int_{0}^{\infty} a(s) \nabla \theta(t-s) d s
$$

* Received October 21, 1969; revised version received April 12, 1970.

1 Gurtin and Pipkin [17] studied temperature-rate waves and computed their speed of propagation. The growth and decay of one-dimensional temperature-rate waves in the nonlinear theory has been discussed by Chen [3]. 
for isotropic materials. Special forms of this linearized theory have proved useful in describing the transmission of heat pulses observed in liquid helium II and some dielectrics at low temperatures (e.g., Brown, Ghung, and Matthews [1]).

In this paper we consider a slightly different memory theory of heat conduction recently proposed by Coleman and Gurtin [7] (see also Gurtin [15]) which does depend on the present value of the temperature gradient. After dispensing with some preliminaries, their results are summarized in Sec. 3. In Sec. 4 we study slow and fast processes and, in each situation, show that the rate of change of the internal energy is approximated by a Gibbs relation and that the corresponding heat flux satisfies a heat conduction inequality. We further show that these inequalities imply that the equilibrium conductivity tensor and the instantaneous conductivity tensor evaluated at the equilibrium history are positive semidefinite.

In Sec. 5 we deduce the linearized theory for isotropic media and show that the heat flux in this instance is given by

$$
\mathbf{q}(t)=-\kappa(0) \nabla \theta(t)-\int_{0}^{\infty} \kappa^{\prime}(s) \nabla \theta(t-s) d s,
$$

where $\kappa(s)$ is the heat conduction relaxation function and $\kappa(0) \geq 0$. Clearly, Eq. (1.5) reduces to Fourier's law (1.1) if $\kappa^{\prime}(s) \equiv 0$; it corresponds to the linearized heat flux relation (1.4) derived by Gurtin and Pipkin [17] if $\kappa(0)=0$.

Secs. 6 and 7 consider further the linearized theory with $\kappa(0)>0$ and $\kappa(0)=0$, respectively. In each case, the appropriate linearized heat equation is cited; a uniqueness theorem is proved for the associated initial-boundary-value problem; and the progressive wave solution is studied. Moreover, for the linearized theory with $\kappa(0)=0$, we indicate the relationship between plane temperature-rate waves ${ }^{2}$ and the corresponding progressive wave solution.

2. Preliminaries. Let us consider a regular region (cf. Kellogg [18]) of three-dimensional Euclidean space $\varepsilon$ occupied by a body $\&$ whose configuration remains unchanged for all time $t \in(-\infty, \infty)$ (i.e., a rigid body). Therefore, we do not distinguish between the material points of $B$ and their spatial positions $x$ in $\varepsilon$.

Fixing our attention to a specific point $x \in \mathcal{B}$, we let $\theta^{t}$ and $\boldsymbol{g}^{t}$, defined by

$$
\theta^{t}(s)=\theta(t-s)=\theta(x, t-s), \quad \boldsymbol{g}^{t}(s)=\boldsymbol{g}(t-s)=\nabla \theta(x, t-s),
$$

$0 \leq s<\infty$, be the histories up to time $t$ of the absolute temperature $(\theta>0)$ and the temperature gradient at $x$. For conciseness we call the ordered pair ${ }^{3}\left(\theta^{t}, g^{t}\right)$ the thermal history $\Lambda^{t}$ at $x$.

Now let us consider a fixed scalar influence function $h(s)$; i.e., a positive, monotonedecreasing, continuous function on $(0, \infty)$ decaying to zero as $s \rightarrow \infty$ fast enough to be square-integrable over $(0, \infty)$. Assuming such a function exists, the norm $\left\|\Lambda^{t}\right\|$ of a measurable function pair $\left(\theta^{t}, g^{t}\right)$ can be defined by

$$
\left\|\Lambda^{t}\right\|^{2}=\left|\Lambda^{t}(0)\right|^{2}+\left.\left\|\Lambda_{r}^{t}\right\|\right|^{2}
$$

${ }^{2}$ Chen [4] has examined the growth and decay of plane waves and waves of arbitrary form within the context of Gurtin and Pipkin's linearized theory.

${ }^{3}$ Henceforth the set of all ordered pairs $\Gamma=(\varphi, v)$, with $\varphi \in R^{+}$(the strictly positive reals) and $\nabla \in \vartheta$ (the inner product space associated with $\varepsilon$ ), will be assumed to have all the properties of the inner product space of dimension four $\Omega^{+} \times \vartheta$ (cf. Coleman [5, p. 10]). 
where

$$
\left\|\Lambda_{r}^{t}\right\|^{2}=\int_{0}^{\infty}\left|\Lambda^{t}(s)\right|^{2} h(s)^{2} d s, \quad\left|\Lambda^{t}(s)\right|^{2}=\Lambda^{t}(s) \cdot \Lambda^{t}(s)
$$

The quantity $\Lambda^{t}(0)=(\theta, g)=\Lambda$ is the present value of $\Lambda^{t}$, and $\Lambda_{r}^{t}=\left(\theta_{r}^{t}, g_{r}^{t}\right)$, called the past history, denotes the restriction of $\Lambda^{t}$ to the open interval $(0, \infty)$. The set of all measurable function pairs $\Lambda^{t}$ with $\left\|\Lambda^{t}\right\|$ finite forms a Hilbert space $\mathcal{H}$. The set of all measurable function pairs $\Lambda_{r}^{t}$ with $\left\|\Lambda_{r}^{t}\right\|$ finite is a subspace of $\mathcal{H C}$ and is denoted by $\mathcal{F}_{r}$.

Throughout this paper, the body $B$ is assumed to be a homogeneous heat conductor with memory characterized at each $x \in B$ by three response functionals $\mathfrak{B}, \mathfrak{N}$, and $\boldsymbol{S}$. These functionals yield the present values of the free energy $\psi(t)$, the entropy $\eta(t)$, and the heat flux $q(t)$ whenever the thermal history $\Lambda^{t}$ is specified at $x$ (cf. Coleman and Gurtin [7, Eqs. (3.3)]):

$$
\begin{aligned}
& \psi=\mathfrak{P}\left(\Lambda^{t}\right), \\
& \eta=\mathfrak{R}\left(\Lambda^{t}\right), \\
& \mathbf{q}=\mathfrak{D}\left(\Lambda^{t}\right) .
\end{aligned}
$$

We take as the common domain of definition of $\mathfrak{P}, \mathfrak{R}$, and $\mathfrak{Q}$ the subset $\mathbb{D}$ in $\mathfrak{F}$ for which $\theta^{\imath}>0$. We further assume that $\mathfrak{B}, \mathfrak{N}$, and $\mathfrak{D}$ are smooth functions over $\mathbb{D}$ with respect to the norm $\left\|\Lambda^{t}\right\|$ in the sense that $\mathfrak{B}$ is twice continuously Fréchet differentiable and $\mathfrak{N}$ and $\mathfrak{Q}$ are each once continuously Fréchet differentiable over $\mathfrak{D}^{4}$

The smoothness assumption for the functional $\mathfrak{B}$ has several well-known consequences. First, it implies the existence and continuity of the first-order differential operators $D_{\Lambda}=\left(D_{\theta}, D_{\mathrm{g}}\right)$ and $\delta_{\Delta}=\left(\delta_{\theta}, \delta_{\mathrm{g}}\right)$ given by (cf. Coleman [6, Sec. 9], Coleman and Gurtin [7, Eqs. (3.9)])

$$
\begin{aligned}
D_{\theta} \mathfrak{P}\left(\Lambda^{t}\right) & =\frac{\partial}{\partial \theta} \mathfrak{P}\left(\theta_{r}^{t}, \mathbf{g}_{r}^{t} ; \theta, \mathbf{g}\right) \\
D_{\mathfrak{g}} \mathfrak{P}\left(\Lambda^{t}\right) \cdot \mathbf{u} & =\left.\frac{d}{d \nu} \mathfrak{P}\left(\theta_{r}^{t}, \mathbf{g}_{\tau}^{t} ; \theta, \mathbf{g}+\nu \mathbf{u}\right)\right|_{\nu=0} \\
\delta_{\theta} \mathfrak{P}\left(\Lambda^{t} \mid \mu_{\tau}\right) & =\left.\frac{d}{d \nu} \mathfrak{P}\left(\theta_{r}^{t}+\nu \mu_{r}, \mathbf{g}_{r}^{t} ; \theta, \mathbf{g}\right)\right|_{\nu=0} \\
\delta_{\mathfrak{g}} \mathfrak{P}\left(\Lambda^{t} \mid \mathbf{u}_{\tau}\right) & =\left.\frac{d}{d \nu} \mathfrak{P}\left(\theta_{r}^{t}, \mathbf{g}_{r}^{t}+\mathbf{u}_{r} ; \theta, \mathbf{g}\right)\right|_{\nu=0}
\end{aligned}
$$

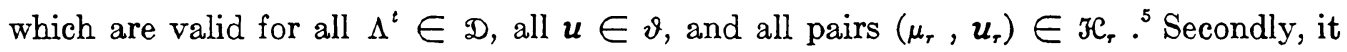
suffices for chain rules (Coleman [5], Mizel and Wang [20]); i.e.,

$$
\dot{\psi}=\frac{d}{d t} \mathfrak{B}\left(\Lambda^{t}\right)=D_{\Lambda} \mathfrak{B}\left(\Lambda^{t}\right) \cdot \dot{\Lambda}+\delta_{\Lambda} \mathfrak{P}\left(\Lambda^{t} \mid \dot{\Lambda}_{r}^{t}\right)
$$

\footnotetext{
- Thus we are assuming that the material obeys the principle of fading memory as formulated by Coleman and Noll [10], [11], [12]. See also Coleman and Mizel [9].

- We note that expressions analogous to (2.5) can also be written for the functionals $\Re$ and $\Omega$ and the functional $\mathcal{F}$ soon to be defined.
} 
whenever the derivatives

$$
\dot{\Lambda}=\frac{d}{d t} \Lambda(t), \quad \dot{\Lambda}_{r}^{t}=\frac{d}{d t} \Lambda(t-s)=-\frac{d}{d s} \Lambda^{t}(s), \quad 0<s<\infty,
$$

exist and are in $\mathcal{F}$. Finally, it implies that the second-order differential operators $D_{\theta}^{2} \mathfrak{B}$, $D_{\theta} \delta_{\theta} \mathfrak{P}=\delta_{\theta} D_{\theta} \mathfrak{P}, \delta_{\theta}^{2} \mathfrak{P}$, etc., exist and are continuous.

Before continuing further, it is appropriate to introduce one other useful parameter; that is, the internal energy $e(t)$ defined by

$$
\begin{aligned}
e & =\psi+\theta \eta, \\
& =\notin\left(\Lambda^{t}\right) .
\end{aligned}
$$

We then define an admissible process in $B$ (at $\mathbf{x}$ ) to be an ordered array $[\theta(\cdot), \mathbf{g}(\cdot)$, $e(\cdot), \eta(\cdot), \mathrm{q}(\cdot)]$ with the following properties:

(i) $\theta(\cdot)$ and $\mathbf{g}(\cdot)$ are continuous and piecewise smooth;

(ii) $\Lambda^{t}=\left(\theta^{t}, \mathbf{g}^{t}\right) \in \mathbb{D}$ for all $t \in(-\infty, \infty)$; and

(iii) $e(\cdot), \eta(\cdot)$, and $\mathrm{q}(\cdot)$ are given by $(2.8)_{1}$ and the constitutive equations (2.4). The functions $e(\cdot)$ and $\mathrm{q}(\cdot)$ determine the heat supply $r(\cdot)$ at $\mathbf{x}$ supplied by the body's external "surroundings" through the energy balance equation

$$
\dot{e}=-\nabla \cdot \mathrm{q}+r \text {. }
$$

In view of the above definition there corresponds to each choice of the time-dependent temperature field $\theta$, a unique admissible process compatible with the balance of energy (cf. Coleman [5, Remark 1]).

3. Consequences of the Clausius-Duhem inequality. By requiring the ClausiusDuhem inequality

$$
\dot{\eta} \geq \frac{r}{\theta}-\nabla \cdot\left(\frac{1}{\theta} \mathrm{q}\right)
$$

to hold for admissible processes, Coleman and Gurtin [7] have shown that the assertions of the next three theorems are true.

THEOREM 3.1. ${ }^{6} \quad$ Assuming that the response functionals $\mathfrak{B}, \mathfrak{R}$, and $\mathfrak{Q}$ obey the hypothesis of fading memory, ${ }^{7}$ the inequality (3.1) is satisfied for all admissible processes if and only if

(i) $D_{\theta} \mathfrak{B}\left(\Lambda^{t}\right)=-\mathfrak{N}\left(\Lambda^{t}\right)$,

(ii) $D_{\mathbf{g}} \mathfrak{P}\left(\Lambda^{t}\right) \equiv 0$, and

(iii) $\mathfrak{Q}\left(\Lambda^{t}\right) \cdot \mathrm{g} \leq \theta^{2} \sigma$ for all sufficiently smooth $\Lambda^{t} \in \mathbb{D}$.

The quantity $\sigma(t)$ is called the internal dissipation and is given by either of the formulae

$$
\begin{aligned}
\sigma(t) & =-\frac{1}{\theta} \delta_{\Lambda} \mathfrak{P}\left(\Lambda^{t} \mid \dot{\Lambda}_{r}^{t}\right), \\
& =-\frac{1}{\theta}(\dot{e}-\theta \dot{\eta}) .
\end{aligned}
$$

An immediate consequence of Theorem 3.1 (i, ii) and (2.8) is that

$$
D_{\theta} \leftleftarrows\left(\Lambda^{t}\right)=\theta D_{\theta} \Re\left(\Lambda^{t}\right)
$$

${ }^{6}$ Cf. Coleman [5, Theorem 1]; Coleman and Gurtin [7, Theorem 6]; Gurtin [15, p. 43].

7 Coleman and Gurtin [7] employed a slightly weaker fading memory hypothesis than that discussed in Sec. 2. 
and

$$
D_{\mathrm{g}} \mathbb{E}\left(\Lambda^{t}\right)=D_{\mathrm{g}} \mathfrak{N}\left(\Lambda^{t}\right) \equiv 0
$$

for every $\Lambda^{t} \in D$.

To examine the equilibrium behavior of the material, we evaluate the response functionals $\mathfrak{B}, \mathfrak{N}, \mathfrak{D}$ and $\mathfrak{E}$ at an equilibrium history $\Lambda_{0}^{\dagger}$; i.e., if $\Lambda^{t}$ is the constant history $\Lambda_{0}^{\dagger}=\left(\theta^{\dagger}, 0^{\dagger}\right)$ where

$$
\theta^{\dagger}(s)=\theta, \quad 0^{\dagger}(s)=0
$$

( 0 is the zero vector in $\vartheta$ ), $0 \leq s<\infty$, then (2.4) and $(2.8)_{2}$ reduce to

$$
\begin{array}{ll}
\psi^{\infty}=\mathfrak{P}\left(\Lambda_{0}^{\dagger}\right)=\hat{\psi}^{\infty}(\theta), & \eta^{\infty}=\mathfrak{N}\left(\Lambda_{0}^{\dagger}\right)=\hat{\eta}^{\infty}(\theta), \\
\mathfrak{q}^{\infty}=\mathfrak{Q}\left(\Lambda_{0}^{\dagger}\right)=\hat{\mathbf{q}}^{\infty}(\theta), \quad e^{\infty}=\psi^{\infty}+\theta \eta^{\infty}=\mathbb{F}\left(\Lambda_{0}^{\dagger}\right)=\hat{e}^{\infty}(\theta) .
\end{array}
$$

Theorem 3.2..$^{8} \quad$ For any $\Lambda^{t} \in D$ with $\theta^{t}(0)=\theta, \mathfrak{B}\left(\Lambda^{t}\right) \geq \hat{\psi}^{\infty}(\theta)$; i.e., of all the thermal histories $\Lambda^{t}$ ending with the present temperature $\theta$, the equilibrium history $\Lambda_{0}^{\dagger}$ results in the least free energy.

This minimal property of the free energy functional $\mathfrak{B}$ necessarily implies that

$$
\begin{gathered}
\delta_{\theta} \mathfrak{P}\left(\Lambda_{0}^{\dagger} \mid \mu_{r}\right)=0, \quad \delta_{\mathbf{g}} \mathfrak{P}\left(\Lambda_{0}^{\dagger} \mid \mathbf{u}_{r}\right)=0 \\
\delta_{\theta}^{2} \mathfrak{P}\left(\Lambda_{0}^{\dagger} \mid \mu_{r}, \mu_{r}\right)+2 \delta_{\theta} \delta_{\mathbf{g}} \mathfrak{P}\left(\Lambda_{0}^{\dagger} \mid \mathbf{u}_{r}, \mu_{r}\right)+\delta_{\mathbf{g}}^{2} \mathfrak{P}\left(\Lambda_{0}^{\dagger} \mid \mathbf{u}_{r}, \mathbf{u}_{r}\right) \geq 0
\end{gathered}
$$

for every $\left(\mu_{r}, \mathrm{u}_{r}\right) \in \mathfrak{H}_{r}$.

THEOREM $3.3 .^{9}$ The equilibrium response functions $\hat{\psi}^{\infty}, \hat{\eta}^{\infty}$, and $\hat{\mathrm{q}}^{\infty}$ obey the equations of classical thermostatics:

$$
\begin{aligned}
& \text { (i) } \frac{d \hat{\psi}^{\infty}}{d \theta}(\theta)=-\hat{\eta}^{\infty}(\theta), \\
& \text { (ii) } \hat{\mathrm{q}}^{\infty}(\theta) \equiv \mathbf{0}
\end{aligned}
$$

for all $\theta \in \mathrm{R}^{+}$.

It follows from (3.6) ${ }_{4}$ and the equilibrium entropy relation (Theorem 3.3 (i)) that

$$
\frac{d \hat{e}^{\infty}}{d \theta}(\theta)=\theta \frac{d \hat{\eta}^{\infty}}{d \theta}(\theta)
$$

In concluding this section, we prove one other result of interest.

Theorem 3.4. Given any equilibrium pair $(\theta, 0)$,

$$
\frac{d \hat{e}^{\infty}}{d \theta}(\theta) \geq D_{\theta} \xi\left(\Lambda_{0}^{\dagger}\right)
$$

i.e., the equilibrium heat capacity is greater than the instantaneous heat capacity evaluated at the equilibrium history $\Lambda_{0}^{\dagger}$.

${ }^{8}$ Cf. Coleman [5, Theorem 3], Coleman and Gurtin [7, Eq. (3.32)], Gurtin [15, p. 45], Gurtin and Pipkin [17, Eq. (4.8)].

- Cf. Coleman and Gurtin [7, Theorem 7], Gurtin [15, p. 45], Gurtin and Pipkin [17, Eqs. (4.11) and (4.12)]. 
Proof. ${ }^{10}$ Since (3.7) is to hold for every $\left(\mu_{r}, \mathbf{u}_{r}\right) \in \mathfrak{F}_{r}$, we let $\mu_{r}$ remain arbitrary and let $\mathbf{u}_{r}=\mathbf{0}_{r}^{\dagger}$, the zero past history. Then, (3.7) reduces to

$$
\delta_{\theta} \mathfrak{P}\left(\Lambda_{0}^{\dagger} \mid \mu_{r}\right)=0, \quad \delta_{\theta}^{2} \mathfrak{B}\left(\Lambda_{0}^{\dagger} \mid \mu_{r}, \mu_{r}\right) \geq 0 .
$$

Now, since (3.9) ${ }_{1}$ holds for all values of $\theta^{\dagger}=\theta 1^{\dagger}\left(1^{\dagger}\right.$ is the constant history with the value of unity),

$$
\frac{d}{d \theta}\left[\delta_{\theta} \mathfrak{B}\left(\Lambda_{0}^{\dagger} \mid \mu_{r}\right)\right]=0 .
$$

Letting $\mu_{r}=1_{r}^{\dagger}$ (the restriction of $1^{\dagger}$ ), (3.10) becomes

$$
\begin{aligned}
\frac{d}{d \theta}\left[\delta_{\theta} \mathfrak{P}\left(\Lambda_{0}^{\dagger} \mid 1_{r}^{\dagger}\right)\right] & =D_{\theta} \delta_{\theta} \mathfrak{P}\left(\Lambda_{0}^{\dagger} \mid 1_{r}^{\dagger}\right)+\delta_{\theta}^{2} \mathfrak{P}\left(\Lambda_{0}^{\dagger} \mid 1_{r}^{\dagger}, 1_{r}^{\dagger}\right), \\
& =-\delta_{\theta} \mathfrak{N}\left(\Lambda_{0}^{\dagger} \mid 1_{r}^{\dagger}\right)+\delta_{\theta}^{2} \mathfrak{P}\left(\Lambda_{0}^{\dagger} \mid 1_{r}^{\dagger}, 1_{r}^{\dagger}\right)=0,
\end{aligned}
$$

where we have made use of the commutative property of $\delta_{\theta}$ and $D_{\theta}$ and the entropy relation (Theorem 3.1 (i)). It follows from $(3.9)_{2}$, with $\mu_{r}=1_{r}^{\dagger}$, and $(3.11)_{2}$ that

$$
\delta_{\theta} \mathfrak{N}\left(\Lambda_{0}^{\dagger} \mid 1_{r}^{\dagger}\right)=\delta_{\theta}^{2} \mathfrak{R}\left(\Lambda_{0}^{\dagger} \mid 1_{r}^{\dagger}, 1_{r}^{\dagger}\right) \geq 0 .
$$

Returning to $(3.6)_{3}$, we compute the derivative of $\hat{\eta}^{\infty}$ with respect to $\theta$ :

$$
\frac{d \hat{\eta}^{\infty}}{d \theta}(\theta)=D_{\theta} \Re\left(\Lambda_{0}^{\dagger}\right)+\delta_{\theta} \Re\left(\Lambda_{0}^{\dagger} \mid 1_{r}^{\dagger}\right),
$$

which, with (3.12), implies that

$$
\frac{d \hat{\eta}^{\infty}}{d \theta}(\theta) \geq D_{\theta} \mathfrak{N}\left(\Lambda_{0}^{\dagger}\right) .
$$

Multiplying this result by $\theta$, and then making use of (3.3) and (3.8), will complete the proof.

Following a customary procedure in thermodynamics, we will assume that

$$
D_{\theta}\left(\Lambda_{0}^{\dagger}\right)>0 \text {. }
$$

4. On slow and fast processes. Let $\theta_{0}$ be a given constant temperature field and let $J\left(\Lambda_{0}\right)$ denote the subset of thermal histories $\Lambda^{t} \in \mathbb{D}$ for which

$$
\Lambda^{t}(s)=\Lambda_{0}^{\dagger}(s)=\left(\theta_{0}^{\dagger}(s), 0^{\dagger}(s)\right)
$$

for every $s \in\left[s_{0}, \infty\right), s_{0}>0 .{ }^{11}$

There corresponds to each $\Lambda^{t} \in J\left(\Lambda_{0}\right)$ a modified thermal history $\Lambda_{(\lambda)}^{t} \in J\left(\Lambda_{0}\right)$ defined by

$$
\Lambda_{(\lambda)}^{t}(s)=\left(\theta_{(\lambda)}^{t}(s), \mathbf{g}_{(\lambda)}^{t}(s)\right)=\left(\theta^{t}(\lambda s), \mathbf{g}^{t}(\lambda s)\right)
$$

$0 \leq s<\infty$, with

$$
\Lambda_{(\lambda)}^{t}(0)=\left(\theta_{(\lambda)}, \mathbf{g}_{(\lambda)}\right)=(\theta, \mathbf{g})=\Lambda
$$

${ }^{10} \mathrm{Cf}$. Coleman [6, Sec. 8], for a similar argument in a different context.

11 This is merely a statement that for some time in the past an equilibrium situation corresponding to the thermal pair $\left(\theta_{0}, 0\right)$ existed in the body $B$. 
We call $\Lambda_{(\lambda)}^{t}$ a retarded history in $J\left(\Lambda_{0}\right)$ if $0<\lambda<1$ (cf. Coleman and Noll [10]) and an accelerated history if $\lambda>1$ (cf. Gurtin and Herrera [16]). A slow process corresponds to the limit $\lambda \rightarrow 0$, a fast process to the limit $\lambda \rightarrow \infty$.

We note for future use that

$$
\frac{d}{d s} \Lambda_{(\lambda)}^{t}(s)=\left.\lambda \frac{d}{d \xi} \Lambda^{t}(\xi)\right|_{\xi-\lambda_{s}}
$$

$0 \leq s<\infty$, and we let $e_{(\lambda)}, \eta_{(\lambda)}, \mathbf{q}_{(\lambda)}$, and $\sigma_{(\lambda)}$ be the internal energy, the entropy, the heat flux, and the internal dissipation at time $t$ corresponding to the thermal history $\Lambda_{(\lambda)}^{t}$.

First, we consider slow processes and in this connection we make use of a lemma due to Coleman and Noll [10].

Lemma 1. Let $\Gamma^{t}()$ be any function in $\Im\left(\Lambda_{0}\right)$. Then, letting $\Gamma_{(\lambda)}^{t}()$ be the retarded history corresponding to $\Gamma^{t}()$ and $\Gamma=\Gamma_{(\lambda)}^{t}(0)$,

where $\Gamma^{\dagger}$ is the constant history with value $\Gamma$.

$$
\lim _{\lambda \rightarrow 0}\left\|\Gamma_{(\lambda)}^{t}()-\Gamma^{\dagger}()\right\|=0
$$

Theorem 4.1. Consider any $\Lambda^{t} \in J\left(\Lambda_{0}\right)$ with $\Lambda^{t}(0)=(\theta, \mathbf{g})$. Then

$$
\begin{aligned}
& \text { (i) }{ }^{12} \lim _{\lambda \rightarrow 0}\left[\dot{e}_{(\lambda)}-\theta \dot{\eta}_{(\lambda)}\right]=0, \\
& \text { (ii) } \mathfrak{\Omega}\left(\Lambda^{\dagger}\right) \cdot \mathrm{g} \leq 0,
\end{aligned}
$$

where $\Lambda^{\dagger}$ is the constant history with the value $\Lambda=(\theta, \mathbf{g})$.

Proof. According to $(3.2)_{1}$, the quantity $\theta_{(\lambda)} \sigma_{(\lambda)}$ is given by

$$
\theta_{(\lambda)} \sigma_{(\lambda)}=-\delta_{\Lambda} \mathfrak{B}\left(\Lambda_{(\lambda)}^{t} \mid \dot{\Lambda}_{(\lambda) r}^{t}\right) \text {. }
$$

Then, by the linearity of $\delta_{\Lambda} \mathfrak{B}$ and Eqs. (2.7) and (4.4), we are permitted to write (4.5) as

$$
\begin{aligned}
\theta_{(\lambda)} \sigma_{(\lambda)} & =\delta_{\Lambda} \mathfrak{P}\left(\Lambda_{(\lambda)}^{t}\left|\lambda \frac{d}{d \xi} \Lambda^{t}(\xi)\right|_{\xi-\lambda_{s}}\right), \\
& =\lambda \delta_{\Lambda} \mathfrak{P}\left(\Lambda_{(\lambda)}^{t}\left|\frac{d}{d \xi} \Lambda^{t}(\xi)\right|_{\xi-\lambda_{s}}\right) .
\end{aligned}
$$

It follows from this, the continuity of $\delta_{\Lambda} \mathfrak{P}$, Lemma 1 , and the boundedness of $\left.(d / d \xi) \Lambda^{t}(\xi)\right|_{\xi-0}$ that (cf. Coleman [5, Remark 14])

$$
\lim _{\lambda \rightarrow 0} \theta_{(\lambda)} \sigma_{(\lambda)}=0 .
$$

Then (3.2) ${ }_{2}$ and (4.3) imply that

$$
\lim _{\lambda \rightarrow 0}-\left[\dot{e}_{(\lambda)}-\theta \dot{\eta}_{(\lambda)}\right]=\lim _{\lambda \rightarrow 0} \theta_{(\lambda)} \sigma_{(\lambda)}=0,
$$

which proves (i). To prove (ii), we first observe that Lemma 1 and the continuity of $\mathfrak{Q}$ imply that

$$
\lim _{\lambda \rightarrow 0} \mathbf{q}_{(\lambda)}=\Omega\left(\Lambda^{\dagger}\right)
$$

${ }^{12}$ Cf. Coleman [5, Remark 15]. It should be pointed out that Coleman's results are stronger than those stated here. 
Then, since the dissipation inequality must hold for all values of $\lambda$ :

$$
\lim _{\lambda \rightarrow 0} \mathbf{q}_{(\lambda)} \cdot \mathbf{g}_{(\lambda)} \leq \lim _{\lambda \rightarrow 0} \theta_{(\lambda)}^{2} \sigma_{(\lambda)} ;
$$

this along with (4.3), (4.7), and (4.9) yields the result desired. Hence Theorem 4.1 implies that during sufficiently slow processes, which start from equilibrium, the rate of change of the internal energy can be approximated by a Gibbs relation and the corresponding heat flux satisfies a heat conduction inequality.

That an analogous result also holds for fast processes will become apparent once we have established

Lemma 2. Let $\Gamma^{t}()$ be any function in $\Im\left(\Lambda_{0}\right)$. Then, letting $\Gamma_{(\lambda)}^{t}()$ be the accelerated history corresponding to $\Gamma^{t}()$ and $\Gamma=\Gamma_{(\lambda)}^{t}(0)$,

$$
\lim _{\lambda \rightarrow \infty}\left\|\Gamma_{(\lambda)}^{t}()-\Gamma^{\ddagger}()\right\|=0
$$

where $\Gamma^{\ddagger}=\Lambda_{0}^{\dagger}$ on $(0, \infty)$ and $\Gamma^{\ddagger}(0)=\Gamma$.

Proof. By definition of the norm (2.2) and (2.3),

$$
\begin{aligned}
\left\|\Gamma_{(\lambda)}^{t}()-\Gamma^{\ddagger}()\right\|^{2}= & \left|\Gamma_{(\lambda)}^{t}(0)-\Gamma^{\ddagger}(0)\right|^{2} \\
& +\int_{0}^{s 0}\left|\Gamma_{(\lambda)}^{t}(s)-\Gamma^{\ddagger}(s)\right|^{2} h(s)^{2} d s \\
& +\int_{s,}^{\infty}\left|\Gamma_{(\lambda)}^{t}(s)-\Gamma^{\ddagger}(s)\right|^{2} h(s)^{2} d s .
\end{aligned}
$$

But, by hypothesis, $\Gamma_{(\lambda)}^{t}(0)=\Gamma^{\ddagger}(0)$; and since $\lambda$ is greater than unity, $\Gamma_{(\lambda)}^{t}(s)=\Gamma^{t}(\lambda s)=$ $\Lambda_{0}^{\dagger}(s)=\Gamma^{\ddagger}(s)$ for all $s \in\left[s_{0}, \infty\right)$. Hence (4.11) reduces to

$$
\left\|\Gamma_{(\lambda)}^{t}()-\Gamma^{\ddagger}()\right\|^{2}=\int_{0}^{s_{0}}\left|\Gamma^{t}(\lambda s)-\Lambda_{0}^{\dagger}(s)\right|^{2} h(s)^{2} d s .
$$

Taking the limit as $\lambda \rightarrow \infty$ and then using Lebesgue's theorem, we have that

$$
\lim _{\lambda \rightarrow \infty}\left\|\Gamma_{(\lambda)}^{t}()-\Gamma^{*}()\right\|^{2}=\int_{0}^{s 0} \lim _{\lambda \rightarrow \infty}\left|\Gamma^{t}(\lambda s)-\Lambda_{0}^{\dagger}(s)\right|^{2} h(s)^{2} d s .
$$

Now for every $\Gamma^{t}(\lambda s) \in J\left(\Lambda_{0}\right), \Gamma^{t}(\lambda s)=\Lambda_{0}^{\dagger}(s)$ for every $\lambda \geq s_{0} / s, 0<s<s_{0}$. Thus, the right side of (4.13) vanishes and the proof is complete.

Theorem 4.2. Consider any $\Lambda^{t} \in J\left(\Lambda_{0}\right)$ with $\Lambda^{t}(0)=(\theta, \mathbf{g})$. Then

$$
\begin{aligned}
& \text { (i) } \lim _{\lambda \rightarrow 0}\left[\dot{e}_{(\lambda)}-\theta \dot{\eta}_{(\lambda)}\right]=0, \\
& \text { (ii) }^{13} \mathfrak{Q}\left(\Lambda^{\dot{*}}\right) \cdot \mathrm{g} \leq 0,
\end{aligned}
$$

where $\Lambda^{\ddagger}=\Lambda_{0}^{\dagger}$ on $(0, \infty)$ and $\Lambda^{\ddagger}(0)=\Lambda=(\theta, \mathbf{g})$.

${ }^{13}$ If the heat flux functional $\Omega$ is independent of the present value of $\mathrm{g}$ (cf. Gurtin and Pipkin $\left[17\right.$, Eq. $\left.(3.6)_{3}\right]$ ) and the material has a center of symmetry, then the limiting behavior for fast processes is adiabatic; i.e., $\lim _{\lambda \rightarrow \infty} q_{(\lambda)}=0$. In this instance, the inequality given is trivially satisfied for every nonzero $\mathrm{g} \in \vartheta$. 
Proof. It is clear that the proof can proceed in the same manner as that for Theorem 4.1 once we have proved that

$$
\lim _{\lambda \rightarrow \infty} \theta_{(\lambda)} \sigma_{(\lambda)}=0 .
$$

Let us examine the quantity $\theta_{(\lambda)} \sigma_{(\lambda)}$ which was given in (4.6) 2 . This expression holds for both retarded and accelerated histories. Therefore, (4.14) can be established by proving that

$$
\delta_{\Delta} \Re\left(\Lambda_{(\lambda)}^{t}\left|\frac{d}{d \xi} \Lambda^{t}(\xi)\right|_{\xi-\lambda_{s}}\right)=0
$$

for all $\lambda$ sufficiently large. Now, for every $\Lambda^{t} \in J\left(\Lambda_{0}\right)$, the derivative $\left.(d / d \xi) \Lambda^{t}(\xi)\right|_{\xi-\lambda_{s}}$ is zero for every $\lambda \geq s_{0} / s, 0<s<s_{0}$, and for every $\lambda>1, s_{0} \leq s<\infty$. Hence, by the continuity and linearity of $\delta_{\perp} \mathfrak{P}$, (4.15) holds and so does (4.14). The proof can now be completed without difficulty.

The inequalities given in Theorems 4.1 and 4.2 are quite similar to the classical heat conduction inequality and it is this similarity which lead us to

Theorem 4.3. The equilibrium conductivity tensor $K(\infty)$ and the instantaneous conductivity tensor $K(0)$ evaluated at the equilibrium history $\Lambda_{0}^{\dagger}=\left(\theta_{0}^{\dagger}, 0^{\dagger}\right)$ are positive semidefinite; i.e., every nonzero $u \in \vartheta$,

$$
\boldsymbol{u} \cdot K(\infty) \boldsymbol{u} \geq 0, \quad \boldsymbol{u} \cdot K(0) \boldsymbol{u} \geq 0,
$$

where

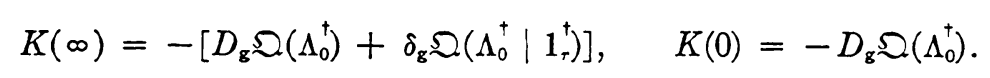

Proof. First consider the inequality

$$
\mathfrak{Q}\left(\Lambda^{\dagger}\right) \cdot g=\mathbb{C}\left(\Lambda^{\dagger}\right) \leq 0 .
$$

Clearly, the functional $\mathfrak{C}$, with $\theta$ arbitrarily fixed, is maximized when $\mathbf{g}=\mathbf{0}$. Let $\theta=\theta_{0}$ and $\mathbf{g}=\xi \mathbf{u}, \mathbf{u}$ any arbitrary nonzero vector in $\vartheta$. Then

$$
\mathfrak{C}\left(\theta_{0} 1_{r}^{\dagger}, \xi \mathrm{u} 1_{r}^{\dagger} ; \theta_{0}, \xi \mathfrak{u}\right)=\mathfrak{Q}\left(\theta_{0} 1_{r}^{\dagger}, \xi \mathfrak{u} 1_{r}^{\dagger} ; \theta_{0}, \xi \mathfrak{u}\right) \cdot \xi \mathbf{u}
$$

and it follows that

$$
\begin{aligned}
& \left.\frac{d}{d \xi} \mathbb{C}\left(\theta_{0} 1^{\dagger}, \xi \mathbf{u} 1_{r}^{\dagger} ; \theta_{0}, \xi \mathbf{u}\right)\right|_{\xi-0}=0, \\
& \left.\frac{d^{2}}{d \xi^{2}} \mathfrak{C}\left(\theta_{0} 1_{r}^{\dagger}, \xi \mathbf{u} 1_{r}^{\dagger} ; \theta_{0}, \xi \mathbf{u}\right)\right|_{\xi-0} \leq 0 .
\end{aligned}
$$

Using (4.17) to compute the derivatives in (4.18), we have that

$$
\mathfrak{Q}\left(\Lambda_{0}^{\dagger}\right) \cdot \mathbf{u}=0,\left.\quad 2 \frac{d}{d \xi} \mathfrak{Q}\left(\theta_{0} 1_{r}^{\dagger}, \xi \mathbf{u} 1_{r}^{\dagger} ; \theta_{0}, \xi \mathbf{u}\right)\right|_{\xi=0} \cdot \mathbf{u} \leq 0 .
$$

The first of (4.19), since it must hold for every nonzero $u \in \vartheta$, yields (see Theorem 3.3(ii))

$$
\mathfrak{Q}\left(\Lambda_{0}^{\dagger}\right)=0 .
$$


Eq. $(4.19)_{2}$, when written in terms of first-order Fréchet differentials, provides us with

$$
\mathbf{u} \cdot\left[D_{\mathbf{z}} \mathfrak{\sim}\left(\Lambda_{0}^{\dagger}\right)+\delta_{\mathbf{g}} \mathfrak{\sim}\left(\Lambda_{0}^{\dagger} \mid 1_{r}^{\dagger}\right)\right] \mathbf{u} \leq 0,
$$

which establishes the nonnegativeness of $K(\infty)$. The restriction on $K(0)$ can be established in an identical manner by considering the inequality

$$
\mathfrak{a}\left(\Lambda^{\ddagger}\right) \cdot \mathrm{g} \leq 0 .
$$

This completes the proof of the theorem.

5. Linearized theory for isotropic materials. Let $\theta_{0}$ be a given constant temperature field. We restrict our consideration here to thermal histories $\Lambda^{t} \in \mathbb{D}$ which are always close to the equilibrium history $\Lambda_{0}^{\dagger}=\left(\theta_{0}^{\dagger}, 0^{\dagger}\right)$ in the sense that

$$
\epsilon=\sup _{\tau \in(-\infty, t)}\left\{\left|\theta(\tau)-\theta_{0}\right|,|g(\tau)|\right\}
$$

is small at each $x \in B$. Then, in view of the assumed smoothness of the response functionals $\mathfrak{O}$ and $\mathfrak{F}$, and Eq. (3.4), the heat flux and the internal energy can be approximated by

$$
\begin{aligned}
& \mathbf{q}=\mathfrak{Q}\left(\Lambda^{t}\right)=\mathfrak{Q}\left(\Lambda_{0}^{\dagger}\right)+D_{\theta} \mathfrak{Q}\left(\Lambda^{t}\right)\left(\theta-\theta_{0}\right)+D_{\mathbf{g}} \mathfrak{Q}\left(\Lambda_{0}^{\dagger}\right) \mathbf{g} \\
& +\delta_{\theta} \cong\left(\Lambda_{0}^{\dagger} \mid \theta_{r}^{t}-\theta_{0} 1_{r}^{\dagger}\right)+\delta_{\mathbf{g}} \cong\left(\Lambda_{0}^{\dagger} \mid \mathbf{g}_{r}^{t}\right)+o(\epsilon), \\
& e=\mathbb{E}\left(\Lambda^{t}\right)=\mathbb{E}\left(\Lambda_{0}^{\dagger}\right)+D_{\theta} \mathbb{E}\left(\Lambda_{0}^{\dagger}\right)\left(\theta-\theta_{0}\right)+\delta_{\theta} \mathbb{E}\left(\Lambda_{0}^{\dagger} \mid \theta_{r}^{t}-\theta_{0} 1_{r}^{\dagger}\right) \\
& +\delta_{\mathbf{g}}\left(\Lambda_{0}^{\dagger} \mid \mathbf{g}_{r}^{t}\right)+o(\epsilon),
\end{aligned}
$$

as $\epsilon \rightarrow 0$. These expressions represent the linearized theory when we neglect the terms of $o(\epsilon){ }^{14}$

We now assume that the material is isotropic. Then the constitutive equations (5.2) must satisfy the identities (cf. Coleman and Mizel [8, Sec. 5])

$$
\mathfrak{Q}\left(\theta^{t} \mathbf{g}^{t}\right)=G^{T} \mathfrak{Q}\left(\theta^{t}, G \mathbf{g}^{t}\right), \quad \mathbb{E}\left(\theta^{t}, \mathbf{g}^{t}\right)=\mathbb{F}\left(\theta^{t}, G \mathbf{g}^{t}\right)
$$

for all orthogonal tensors $G \in \mathcal{O}$, the full orthogonal group. Since $-I \in \mathcal{O}$ ( $I$ is the unit tensor), it follows from (5.3) that

$$
\mathfrak{Q}\left(\theta^{t}, \mathbf{0}^{\dagger}\right)=0, \quad \mathfrak{F}\left(\theta^{t}, \mathbf{g}^{t}\right)=\mathbb{F}\left(\theta^{t},-\mathbf{g}^{t}\right),
$$

which in turn implies that

$$
D_{\theta} \mathfrak{Q}\left(\Lambda_{0}^{\dagger}\right)=0, \quad \delta_{\theta} \mathfrak{Q}\left(\Lambda_{0}^{\dagger} \mid \theta_{r}^{t}-\theta_{0} 1_{r}^{\dagger}\right)=0, \quad \delta_{\mathrm{g}} \mathbb{E}\left(\Lambda_{0}^{\dagger} \mid \mathbf{g}_{r}^{t}\right)=0 .
$$

These results (5.5) plus the fact that $\mathfrak{Q}\left(\Lambda_{0}^{\dagger}\right)=0$ from Theorem 3.3 (ii), reduces (5.2) to

$$
\mathbf{q}=D_{\mathbf{g}} \mathfrak{Q}\left(\Lambda_{0}^{\dagger}\right) \mathbf{g}+\delta_{\mathbf{g}} \mathfrak{S}\left(\Lambda_{0}^{\dagger} \mid \mathbf{g}_{r}^{t}\right), \quad e=e_{0}+D_{\theta} \mathbb{E}\left(\Lambda_{0}^{\dagger}\right) \theta+\delta_{\theta} \mathbb{E}\left(\Lambda_{0}^{\dagger} \mid \theta_{r}^{t}\right),
$$

where $e_{0}$ is a constant defined by

$$
e_{0}=\mathbb{E}\left(\Lambda_{0}^{\dagger}\right)-D_{\theta} \mathbb{E}\left(\Lambda_{0}^{\dagger}\right) \theta_{0}-\delta_{\theta} \mathbb{E}\left(\Lambda_{0}^{\dagger} \mid \theta_{0} 1_{\tau}^{\dagger}\right) .
$$

The mapping $\mathbf{g}_{r}^{t} \rightarrow \delta_{\mathrm{g}} \mathfrak{Q}\left(\Lambda_{0}^{\dagger} \mid \mathbf{g}_{r}^{t}\right)$ is bounded and linear in $\mathbf{g}_{r}^{t}$ and it maps the Hilbert space $\mathcal{H}_{r}$ into $\vartheta$. Then by the Riesz representation theorem $\delta_{\mathrm{g}} \mathfrak{\Omega}\left(\Lambda_{0}^{\dagger} \mid \mathbf{g}_{r}^{t}\right)$ can be represented as an inner product on $\mathfrak{F}_{r}$ for every $\mathbf{g}_{r}^{t} \in \mathfrak{F}_{r}$; i.e.,

\footnotetext{
14 We do not develop the linearized constitutive equations for the free energy or entropy as they are not needed in the sequel.
} 


$$
\delta_{\mathbf{g}} \mathfrak{O}\left(\Lambda_{0}^{\dagger} \mid \mathbf{g}_{r}^{t}\right)=\int_{0}^{\infty} T(s) \mathbf{g}^{t}(s) h(s)^{2} d s,
$$

where $T$ is a uniquely defined tensor-valued function on $(0, \infty)$ with components in $\mathfrak{F}_{r}$. Clearly, $T$ depends on $\theta_{0}$ as well as $s$. Now let $K(s), 0 \leq s<\infty$, be the unique solution of

$$
K^{\prime}(s)=\frac{d}{d s} K(s)=-T(s) h(s)^{2}, \quad K(0)=-D_{\mathrm{g}} \mathfrak{Q}\left(\Lambda_{0}^{\dagger}\right) .
$$

By the isotropy of the material, $K(s)$ must be an isotropic tensor:

$$
K(s)=\kappa(s) I .
$$

Hence, combining (5.8)-(5.10) with $(5.6)_{1}$ we arrive at the linearized constitutive equation for the heat flux:

$$
\mathrm{q}=-\kappa(0) \mathrm{g}-\int_{0}^{\infty} \kappa^{\prime}(s) \mathrm{g}(t-s) d s .
$$

The scalar-valued function $\kappa(s)$ is called the heat conduction relaxation function and $\kappa(0)$ the instantaneous conductivity. If $\mathbf{g}(t-s)=\mathbf{g}_{0}^{\dagger}(s)=\mathbf{g}_{0}$, a constant vector in $\vartheta$, for all $s \in[0, \infty)$, then either $(5.6)_{1}$ or $(5.11)$ will yield the steady-state heat flux $\mathbf{q}_{0}$ :

$$
\mathrm{q}_{0}=-\kappa(\infty) \mathrm{g}_{0} \text {, }
$$

where $\kappa(\infty)$ is the equilibrium conductivity. It follows directly from Theorem 4.3 that both $\kappa(\infty)$ and $\kappa(0)$ are nonnegative. While we would expect in most physical situations that the equilibrium conductivity $\kappa(\infty)$ would be nonzero, there is no such justification for assuming that the instantaneous conductivity $\kappa(0)$ also be nonzero. In fact, in view of the experimental evidence cited earlier with regard to the linearized theory derived by Gurtin and Pipkin [17], there is reason to suspect that in some materials $\kappa(0)$ may become zero at very low reference temperatures $\theta_{0}$.

Proceeding in an analogous fashion, we can obtain from $(5.6)_{2}$ the linearized constitutive equation for the internal energy:

$$
e=e_{0}+\alpha(0) \theta+\int_{0}^{\infty} \alpha^{\prime}(s) \theta(t-s) d s
$$

where

$$
\alpha(0)=D_{\theta} \mathbb{E}\left(\Lambda_{0}^{\dagger}\right), \quad \alpha(\infty)=\left[D_{\theta} \mathbb{E}\left(\Lambda_{0}^{\dagger}\right)+\delta_{\theta} \mathbb{E}\left(\Lambda_{0}^{\dagger} \mid 1_{\tau}^{\dagger}\right)\right]=\frac{d \hat{e}^{\infty}}{d \theta}\left(\theta_{0}\right) .
$$

The function $\alpha(s)$ is called the energy-temperature relaxation function with $\alpha(0)$ being the instantaneous heat capacity and $\alpha(\infty)$ the equilibrium heat capacity. Theorem $3.4 \mathrm{im}-$ plies that

$$
\alpha(\infty) \geq \alpha(0)
$$

and following (3.15), we assume $\alpha(0)>0$.

To avoid repeated comments regarding the smoothness properties of the relaxation functions $\kappa(s)$ and $\alpha(s)$, we assume once and for all that they are each of class $C^{4}$ on $[0, \infty)$ and that the first four of their derivatives are bounded and absolutely integrable on $[0, \infty)$. It follows from the Riesz theorem that the first derivatives $\kappa^{\prime}(s)$ and $\alpha^{\prime}(s)$ have the properties 


$$
\int_{0}^{\infty}\left|\kappa^{\prime}(s)\right|^{2} h(s)^{-2} d s<\infty, \quad \int_{0}^{\infty}\left|\alpha^{\prime}(s)\right|^{2} h(s)^{-2} d s<\infty .
$$

This property (5.16) implies that the functions $\kappa^{\prime}(s)$ and $\alpha^{\prime}(s)$ must approach zero at a faster rate than the influence function $h(s)$ as $s \rightarrow \infty$. Henceforth, we shall assume that functions $\kappa^{\prime \prime}(s), \alpha^{\prime \prime}(s), \kappa^{\prime \prime \prime}(s)$ and $\alpha^{\prime \prime \prime}(s)$ also have this property.

6. Linearized theory with nonvanishing $\kappa(0)$. In this case, Eqs. (5.11), (5.13), and (2.9) provide us with a complete set of field equations and they can be combined, noting that $\mathrm{g}=\nabla \theta$, to give the linearized heat equation:

$$
\begin{aligned}
\alpha(0) \dot{\theta}(\mathbf{x}, t) & +\int_{0}^{\infty} \alpha^{\prime}(s) \dot{\theta}(\mathbf{x}, t-s) d s=\kappa(0) \nabla^{2} \theta(\mathbf{x}, t) \\
& +\int_{0}^{\infty} \kappa^{\prime}(s) \nabla^{2} \theta(\mathbf{x}, t-s) d s+r(\mathbf{x}, t) .
\end{aligned}
$$

(a) A uniqueness theorem. Here our concern is with obtaining sufficient conditions for the uniqueness of solutions to the initial-boundary-value problem associated with (6.1).

Due to the linearity of the field equations, it clearly suffices to show that null initial and boundary data will yield a null solution. that $^{15}$

TheORem 6.1. Let $\theta$ be a solution of (6.1) on $B \times(-\infty, \infty)$. Furthermore, suppose

$$
\alpha(0)>0, \quad \alpha^{\prime}(0)>0, \quad \kappa(0)>0 ;
$$

and

$$
\begin{aligned}
& \theta=0 \quad \text { on } B \times(-\infty, 0], \quad r=0 \quad \text { on } B \times(-\infty, \infty), \\
& \theta=0 \quad \text { on } d B_{\theta} \times(0, \infty), \quad \mathrm{q} \cdot \mathbf{n}=0 \quad \text { on } d B_{\mathbf{g}} \times(0, \infty),
\end{aligned}
$$

where $d \Theta^{\prime}=d \Theta_{\theta} \cup d \Theta_{\mathbf{q}}$. Then,

$$
\theta \equiv 0 \quad \text { on } B \times(-\infty, \infty) .
$$

Proof. Let us compute the quantity ${ }^{16}$

$$
S_{1}(t)=\int_{-\infty}^{t} \int_{d \Theta} \dot{\theta} \mathbf{q} \cdot \mathbf{n} d A d \tau-\int_{-\infty}^{t} \int_{\mathbb{B}} \dot{\theta} r d V d \tau .
$$

Application of the divergence theorem and the energy balance equation (2.9) to (6.2) vields

$$
S_{1}(t)=-\int_{-\infty}^{t} \int_{B}(\dot{\theta} \dot{e}-\mathbf{q} \cdot \dot{\mathbf{g}}) d V d \tau .
$$

Using Fubini's theorem to interchange the order of integration and the linearized constitutive equations (5.11) and (5.13), we have

\footnotetext{
15 We believe that $\alpha^{\prime}(0)>0$ will insure that $\theta(\mathbf{x}, t)$ is asymptotically stable in the sense that $\lim _{t \rightarrow \infty}$ $\theta(\mathbf{x}, t)=f(\mathbf{x}) ; f(\mathbf{x})$ finite.

${ }^{16}$ We might call $S_{1}(t)$ the thermal work; cf. Day and Gurtin [13, Eq. (6)].
} 


$$
\begin{aligned}
s_{1}(t)= & -\int_{B} \int_{-\infty}^{t} \alpha(0) \dot{\theta}(\mathbf{x}, \tau)^{2} d \tau d V-\int_{B} \int_{-\infty}^{t} \alpha^{\prime}(0) \theta(\mathbf{x}, \tau) \dot{\theta}(\mathbf{x}, \tau) d \tau d V \\
& -\int_{B} \int_{-\infty}^{t}\left(\int_{0}^{\infty} \alpha^{\prime \prime}(s) \theta(\mathbf{x}, \tau-s) d s\right) \dot{\theta}(\mathbf{x}, \tau) d \tau d V \\
& -\int_{B} \int_{-\infty}^{t} \kappa(0) \mathbf{g}(\mathbf{x}, \tau) \cdot \dot{\mathbf{g}}(\mathbf{x}, \tau) d \tau d V \\
& -\int_{B} \int_{-\infty}^{t}\left(\int_{0}^{\infty} \kappa^{\prime}(s) \mathbf{g}(\mathbf{x}, \tau-s) d s\right) \cdot \dot{\mathbf{g}}(\mathbf{x}, \tau) d \tau d V .
\end{aligned}
$$

If we now institute a change of variables $(\rho=\tau-s)$ in the third and fifth terms and then integrate these expressions by parts, the resulting expression for $S_{1}(t)$ is

$$
\begin{aligned}
S_{1}(t)= & -\int_{\mathbb{B}} \int_{-\infty}^{t} \alpha(0) \dot{\theta}(\mathbf{x}, \tau)^{2} d \tau d V-\int_{\mathbb{B}} \frac{\alpha^{\prime}(0)}{2} \theta(\mathbf{x}, t)^{2} d V \\
& -\int_{\mathbb{B}} \int_{-\infty}^{t} \alpha^{\prime \prime}(t-\tau) \theta(\mathbf{x}, t) \theta(\mathbf{x}, \tau) d \tau d V+\int_{\mathbb{B}} \int_{-\infty}^{t} \alpha^{\prime \prime}(0) \theta(\mathbf{x}, \tau)^{2} d \tau d V \\
& +\int_{\mathbb{B}} \int_{-\infty}^{t} \int_{-\infty}^{\tau} \alpha^{\prime \prime \prime}(\tau-\rho) \theta(\mathbf{x}, \tau) \theta(\mathbf{x}, \rho) d \rho d \tau d V \\
& -\int_{\mathbb{B}} \frac{\kappa(0)}{2} \mathbf{g}(\mathbf{x}, t) \cdot \mathbf{g}(\mathbf{x}, t) d V-\int_{\mathbb{B}} \int_{-\infty}^{t} \kappa^{\prime}(t-\tau) \mathbf{g}(\mathbf{x}, t) \cdot \mathbf{g}(\mathbf{x}, \tau) d \tau d V \\
& +\int_{\mathbb{B}} \int_{-\infty}^{t} \kappa^{\prime}(0) \mathbf{g}(\mathbf{x}, \tau) \cdot \mathbf{g}(\mathbf{x}, \tau) d \tau d V \\
& +\int_{\mathbb{B}} \int_{-\infty}^{t} \int_{-\infty}^{\tau} \kappa^{\prime \prime}(\tau-\rho) \mathbf{g}(\mathbf{x}, \tau) \cdot \mathbf{g}(\mathbf{x}, \rho) d \rho d \tau d V .
\end{aligned}
$$

The initial condition $\theta=0$ on $B \times(-\infty, 0]$ permits the lower limits in $(6.5)$ to be changed to zero and the remaining null data implies that $S_{1}(t) \equiv 0$ for every $t \in(0, \infty)$.

For convenience we now set

and

$$
\varphi=\theta\left(\alpha^{\prime}(0) / 2\right)^{1 / 2}, \quad \nabla=\mathbf{g}(\kappa(0) / 2)^{1 / 2}
$$

$$
\gamma_{1}(t-\tau)=2 \alpha^{\prime \prime}(t-\tau) / \alpha^{\prime}(0), \quad \gamma_{2}(t-\tau)=2 \kappa^{\prime}(t-\tau) / \kappa(0) .
$$

Since, by hypothesis, the functions $\alpha$ and $\kappa$ are of class $C^{4}$ on $[0, \infty)$, there exists a bound $B\left(t_{0}\right)$ such that

$$
B\left(t_{0}\right)=\sup _{t \in\left(0, t_{0}\right)}\left\{\left|\gamma_{1}(t)\right|+\left|\gamma_{2}(t)\right|+\left|\gamma_{1}^{\prime}(t)\right|+\left|\gamma_{2}^{\prime}(t)\right|\right\}<\infty
$$

for every $t_{0} \in(0, \infty)$. Let us suppose that $t_{0}$ has been arbitrarily fixed and that $t \leq t_{0}$. Then, using (6.6)-(6.8), and letting $\Phi$ be the ordered pair $(\varphi, \nabla)$, we can obtain the following inequality from (6.5):

$$
\begin{gathered}
\int_{B} \int_{0}^{t} \alpha(0)|\dot{\theta}(\mathbf{x}, \tau)|^{2} d \tau d V+\int_{B}|\Phi(\mathbf{x}, t)|^{2} d V \\
\leq B\left(t_{0}\right)\left\{\int_{B} \int_{0}^{t}|\Phi(\mathbf{x}, \tau)||\Phi(\mathbf{x}, t)| d \tau d V+\int_{B} \int_{0}^{t}|\Phi(\mathbf{x}, \tau)|^{2} d \tau d V\right. \\
\left.+\int_{B} \int_{0}^{t} \int_{0}^{\tau}|\Phi(\mathbf{x}, \tau)||\Phi(\mathbf{x}, \rho)| d \rho d \tau d V\right\} .
\end{gathered}
$$


Both terms on the left side of the inequality are nonnegative and bounded, and hence it follows that

$$
\begin{aligned}
\int_{B}|\Phi(\mathbf{x}, t)|^{2} d V \leq B\left(t_{0}\right)\left\{\int_{B} \int_{0}^{t}|\Phi(\mathbf{x}, \tau)| \mid \Phi(\mathbf{x}, t) d \tau d V\right. \\
\left.\quad+\int_{B} \int_{0}^{t}|\Phi(\mathbf{x}, \tau)|^{2} d \tau d V+\int_{B} \int_{0}^{t} \int_{0}^{\tau}|\Phi(\mathbf{x}, \tau)||\Phi(\mathbf{x}, \rho)| d \rho d \tau d V\right\} .
\end{aligned}
$$

That this inequality implies the null solution $\Phi \equiv 0$ on $B \times(0, \infty)$ follows almost verbatim an argument given by Edelstein and Gurtin [14, proof of Lemma 3] to establish a similar result. Hence the proof is complete.

(b) Plane progressive waves. Consider the time-dependent temperature field

$$
\theta(\mathbf{x}, t)-\theta_{0}=A \operatorname{Re}\left\{\exp (-\xi \mathbf{p} \cdot \mathbf{m}) \exp \left(i \omega\left(t-\frac{1}{u} \mathbf{p} \cdot \mathbf{m}\right)\right)\right\}, \quad \mathbf{p}=\mathbf{x}-\mathbf{x}_{0},
$$

for $\omega>0, \xi \geq 0, u \geq 0$, and $A$, all real numbers. The function (6.11) is called a damped plane progressive wave with frequency $\omega$, attenuation $\xi$, speed $u$, direction $\mathbf{m}(|\mathbf{m}|=1)$, and amplitude $A$. Clearly, this temperature field satisfies the linearized heat equation (6.1) (with $r=0$ ) if and only if

$$
i \omega\left\{\alpha(0)+\bar{\alpha}^{\prime}(\omega)\right\}=(\xi+i \omega / u)^{2}\left\{\kappa(0)+\bar{\kappa}^{\prime}(\omega)\right\}
$$

where $\bar{\alpha}^{\prime}(\omega)$ and $\bar{\kappa}^{\prime}(\omega)$ are Fourier transforms:

$$
\bar{\alpha}^{\prime}(\omega)=\int_{0}^{\infty} \alpha^{\prime}(s) e^{-i \omega s} d s, \quad \bar{\kappa}^{\prime}(\omega)=\int_{0}^{\infty} \kappa^{\prime}(s) e^{-i \omega \cdot} d s .
$$

Then, for each frequency $\omega$ there is exactly one set of values $u=u(\omega) \geq 0, \xi=\xi(\omega) \geq 0$ which satisfies (6.12):

$$
\begin{aligned}
u(\omega)^{2} & =\frac{2 \omega\left|\kappa(0)+\bar{\kappa}^{\prime}(\omega)\right|}{\left|\alpha(0)+\bar{\alpha}^{\prime}(\omega)\right|}[\sec (\nu(\omega)-\varphi(\omega))][\tan (\nu(\omega)-\varphi(\omega))+\sec (\nu(\omega)-\varphi(\omega))], \\
\xi(\omega) & =\frac{\omega}{u(\omega)}[\tan (\nu(\omega)-\varphi(\omega))+\sec (\nu(\omega)-\varphi(\omega))],
\end{aligned}
$$

where

$$
\tan \nu(\omega)=\frac{\operatorname{Im}\left(\kappa(0)+\bar{\kappa}^{\prime}(\omega)\right)}{\operatorname{Re}\left(\kappa(0)+\bar{\kappa}^{\prime}(\omega)\right)}, \quad \tan \varphi(\omega)=\frac{\operatorname{Im}\left(\alpha(0)+\bar{\alpha}^{\prime}(\omega)\right)}{\operatorname{Re}\left(\alpha(0)+\bar{\alpha}^{\prime}(\omega)\right)} .
$$

For classical heat conduction $\alpha(s)=\alpha(\infty)$ (constant), $k(s)=\kappa(\infty)$ (constant) for every $s \in[0, \infty)$ and $(6.14)$ yields

$$
u_{c}(\omega)=\left(\frac{2 \omega \kappa(\infty)}{\alpha(\infty)}\right)^{1 / 2}, \quad \xi_{c}(\omega)=\left(\frac{\omega \alpha(\infty)}{2 \kappa(\infty)}\right)^{1 / 2} .
$$

It follows from (6.13), and some known theorems on Fourier transforms that

$$
\lim _{\omega \rightarrow 0} \bar{\alpha}^{\prime}(\omega)=\alpha(\infty)-\alpha(0), \quad \lim _{\omega \rightarrow 0} \omega \bar{\alpha}^{\prime}(\omega)=0
$$

and

$$
\lim _{\omega \rightarrow \infty} \bar{\alpha}^{\prime}(\omega)=0, \quad \lim _{\omega \rightarrow \infty} \omega \bar{\alpha}^{\prime}(\omega)=-i \alpha^{\prime}(0) .
$$


Similar limits can also be obtained for $\bar{\kappa}^{\prime}(\omega)$. Then, using these formulas in connection with (6.14), it is not difficult to show that as $\omega \rightarrow 0$

$$
u(\omega) \rightarrow u_{c}(\omega), \quad \xi(\omega) \rightarrow \xi_{c}(\omega),
$$

and as $\omega \rightarrow \infty$

$$
u(\omega) \rightarrow(2 \omega \kappa(0) / \alpha(0))^{1 / 2}, \quad \xi(\omega) \rightarrow(\omega \alpha(0) / 2 \kappa(0))^{1 / 2} .
$$

Thus, we see that for low frequencies $(\omega \rightarrow 0)$ the speed and attenuation of a damped plane progressive wave approach the values predicted by the classical theory. For high frequencies $(\omega \rightarrow \infty)$, the wave speed and attenuation are real and unbounded as in the classical theory. Moreover, for $\kappa(0) / \alpha(0)$ greater (less) than $\kappa(\infty) / \alpha(\infty)$, the wave speed is greater (less) than and the attenuation is less (greater) than the values predicted by the classical theory.

7. Linearized theory with vanishing $\kappa(0)$. For this case the linearized constitutive equation for the heat flux becomes

$$
\mathbf{q}=-\int_{0}^{\infty} \kappa^{\prime}(s) \mathbf{g}(t-s) d s
$$

and the linearized heat equation (6.1) reduces to

$$
\alpha(0) \dot{\theta}(\mathbf{x}, t)+\int_{0}^{\infty} \alpha^{\prime}(s) \dot{\theta}(\mathbf{x}, t-s) d s=\int_{0}^{\infty} \kappa^{\prime}(s) \nabla^{2} \theta(\mathbf{x}, t-s) d s+r(\mathbf{x}, t) .
$$

(a) A uniqueness theorem. As before, we are concerned with obtaining sufficient conditions for the uniqueness of solutions to the initial-boundary-value problem associated with the heat equation (7.2).

Theorem 7.1. Let $\theta$ be a solution of (7.2) on $B \times(-\infty, \infty)$. Furthermore, suppose that $^{18}$

$$
\alpha(0)>0, \quad \alpha^{\prime}(0) \geq 0, \quad \alpha^{\prime \prime}(0)>0, \quad \kappa^{\prime}(0)>0,
$$

and

$$
\begin{aligned}
& \theta=0 \quad \text { on } \quad \beta \times(-\infty, 0 \mathrm{~J}, \quad r=0 \quad \text { on } \quad \beta \times(-\infty, \infty), \\
& \theta=0 \quad \text { on } \quad d 03_{\theta} \times(0, \infty), \quad \mathbf{q} \cdot \mathbf{n}=0 \quad \text { on } \quad d \Theta_{\mathbf{q}} \times(0, \infty) ;
\end{aligned}
$$

where $d B_{B}=d B_{\theta} \cup d B_{\mathbb{a}}$. Then

$$
\theta \equiv 0 \text { on } B \times(-\infty, \infty) .
$$

The proof of this result is omitted since it is very similar to the one given for Theorem 6.1 once the quantity

$$
S_{2}(t)=\int_{-\infty}^{t} \int_{d B} \dot{\theta} \dot{\mathrm{q}} \cdot \mathbf{n} d A d \tau-\int_{-\infty}^{t} \int_{B} \dot{\theta} \dot{r} d V d \tau .
$$

has been computed.

${ }^{17}$ This equation can be written in a form similar to that derived by Gurtin and Pipkin [17]:

$$
\alpha(0) \ddot{\theta}+\alpha^{\prime}(0) \dot{\theta}+\int_{0}^{\infty} \alpha^{\prime \prime}(s) \dot{\theta}(t-s) d s=\kappa^{\prime}(0) \nabla^{2} \theta+\int_{0}^{\infty} \kappa^{\prime \prime}(s) \nabla^{2} \theta(t-s) d s+\dot{r}
$$
stable.

${ }^{18}$ The positiveness of $\alpha^{\prime}(0)$ and $\alpha^{\prime \prime}(0)$ is believed to insure that the solution $\theta(x, t)$ is asymptotically 
(b) Plane progressive waves. Let us again consider the sinusoidal temperature field (6.11). Then the heat equation (7.2) (with $r=0$ ) implies that the wave speed $u$ and the attenuation $\xi$ must obey the relations

$$
\begin{aligned}
u(\omega)^{2} & =\frac{2 \omega\left|\bar{\kappa}^{\prime}(\omega)\right|}{\left|\alpha(0)+\bar{\alpha}^{\prime}(\omega)\right|}[\sec (\nu(\omega)-\varphi(\omega))][\tan (\nu(\omega)-\varphi(\omega))+\sec (\nu(\omega)-\varphi(\omega))], \\
\xi(\omega) & =\frac{\omega}{u(\omega)}[\tan (\nu(\omega)-\varphi(\omega))+\sec (\nu(\omega)-\varphi(\omega))],
\end{aligned}
$$

where

$$
\tan \nu(\omega)=\frac{\operatorname{Im}\left(\bar{\kappa}^{\prime}(\omega)\right)}{\operatorname{Re}\left(\bar{\kappa}^{\prime}(\omega)\right)}, \quad \tan \varphi(\omega)=\frac{\operatorname{Im}\left(\alpha(0)+\bar{\alpha}^{\prime}(\omega)\right)}{\operatorname{Re}\left(\alpha(0)+\bar{\alpha}^{\prime}(\omega)\right)} .
$$

Moreover, using the limits (6.17) and their counterparts for $\bar{\kappa}^{\prime}(\omega)$, it is not difficult to verify that as $\omega \rightarrow 0, u$ and $\xi$ approach the classical values $u_{c}$ and $\xi_{c}$.

To analyze (7.4) for large $\omega$, an appropriate asymptotic expansion for each of the Fourier transforms $\bar{\alpha}^{\prime}(\omega)$ and $\bar{\kappa}^{\prime}(\omega)$ is required and can be obtained by integrating (6.13) twice by parts:

$$
\begin{aligned}
& \bar{\alpha}^{\prime}(\omega)=-\frac{\alpha^{\prime}(0) i}{\omega}-\frac{\alpha^{\prime \prime}(0)}{\omega^{2}}-\frac{\bar{\alpha}^{\prime \prime \prime}(\omega)}{\omega^{2}}, \\
& \bar{\kappa}^{\prime}(\omega)=-\frac{\kappa^{\prime}(0) i}{\omega}-\frac{\kappa^{\prime \prime}(0)}{\omega^{2}}-\frac{\bar{\kappa}^{\prime \prime \prime}(\omega)}{\omega^{2}},
\end{aligned}
$$

where $\bar{\alpha}^{\prime \prime \prime}(\omega)$ and $\bar{\kappa}^{\prime \prime \prime}(\omega)$ are the Fourier transforms of $\alpha^{\prime \prime \prime}(s)$ and $\kappa^{\prime \prime \prime}(s)$. We assume that $\alpha(0)>0, \alpha^{\prime}(0) \geq 0, \alpha^{\prime \prime}(0)>0$, and $\kappa^{\prime}(0)>0$ to insure that the temperature field (6.11) is unique. It also seems physically reasonable to assume $\kappa^{\prime \prime}(0)<0$ on the basis of some statistical arguments given by Maxwell [19] and Cattaneo [2]. Then, as $\omega \rightarrow \infty$,

$$
u(\omega) \rightarrow\left(\frac{\kappa^{\prime}(0)}{\alpha(0)}\right)^{1 / 2}, \quad \xi(\omega) \rightarrow-\frac{1}{2}\left(\frac{\kappa^{\prime \prime}(0)}{\kappa^{\prime}(0)}-\frac{\alpha^{\prime}(0)}{\alpha(0)}\right)\left(\frac{\alpha(0)}{\kappa^{\prime}(0)}\right)^{1 / 2} .
$$

These results are quite different from those obtained for the linearized theory with $\kappa(0)>0$ in that now the wave speed and attenuation remain finite for arbitrarily large $\omega$.

(c) Plane temperature-rate waves. As has already been indicated, there can exist, in the linearized theory with $\kappa(0)=0$, jump discontinuities in the "temperature-rate" field $\dot{\theta}$ which propagate with a finite speed. Using a singular surface analysis (cf. Truesdell and Toupin [21, Sec. C]) and assuming the wave to be plane, we find that the propagation of such waves, in terms of our present study, is governed by ${ }^{19}$

THEOREM 7.2. Consider a plane temperature-rate wave propagating into an infinite heat conductor with $\kappa(0)=0$. Then, for $t \geq 0$, the speed $U$ is a constant given by ${ }^{20}$

$$
U=\left(\frac{\kappa^{\prime}(0)}{\alpha(0)}\right)^{1 / 2}
$$

and the amplitude $a(a=[\dot{\theta}])^{21}$ has the following dependence on $t::^{22}$

${ }^{19}$ Here the definition of an admissible process has been extended to include the functions $\mathrm{g}(\cdot)$ which are piecewise continuous.

${ }^{20}$ Gurtin and Pipkin [17].

21 " [- ]" denotes the jump; Truesdell and Toupin [21].

${ }^{22}$ Chen [4]. 


$$
a(t)=a(0) \exp \left[\frac{1}{2}\left(\frac{\kappa^{\prime \prime}(0)}{\kappa^{\prime}(0)}-\frac{\alpha^{\prime}(0)}{\alpha(0)}\right) t\right] .
$$

Clearly, in view of the sufficient conditions for unique solutions of initial-boundaryvalue problems and the assumption of $\kappa^{\prime \prime}(0)<0$, the wave speed is real and the amplitude of the wave decays monotonically to zero as $t \rightarrow \infty$.

Our primary interest here is in the relationship between plane temperature-rate waves and the plane progressive waves discussed earlier. Let us define the ultrasonic speed $u_{\infty}$ and the ultrasonic attenuation $\xi_{\infty}$ by

$$
u_{\infty}=\lim _{\omega \rightarrow \infty} u(\omega), \quad \xi_{\infty}=\lim _{\omega \rightarrow \infty} \xi(\omega) .
$$

Then (7.7) implies that

$$
u_{\infty}=\left(\frac{\kappa^{\prime}(0)}{\alpha(0)}\right)^{1 / 2}, \quad \xi_{\infty} u_{\infty}=-\frac{1}{2}\left(\frac{\kappa^{\prime \prime}(0)}{\kappa^{\prime}(0)}-\frac{\alpha^{\prime}(0)}{\alpha(0)}\right) .
$$

Setting this result alongside Theorem 7.2, we arrive at

THEOREM 7.3. Let $U$ and $a(t)$ be the speed and amplitude of a plane temperature-rate wave which since $t=0$ has been propagating into an infinite heat conductor with $\kappa(0)=0$. Then, for $t \geq 0 \quad U=u_{\infty}$ and

$$
a(t)=a(0) \exp \left(-u_{\infty} \xi_{\infty} t\right)
$$

where $u_{\infty}$ and $\xi_{\infty}$ are the ultrasonic speed and attenuation of damped plane progressive waves in the same body.

Acknowledgements. This paper, in a somewhat different form, was submitted to the Graduate School of West Virginia University as the author's doctoral dissertation. The author takes special pleasure in acknowledging his advisor, Professor Robert D. Snyder, for his helpful guidance. The author would also like to express his thanks to Dr. Peter J. Chen and Professor Morton E. Gurtin for many valuable discussions and for their critical comments regarding an earlier draft of this manuscript.

During the course of this investigation the author had the financial support of the National Aeronautics and Space Administration and the West Virginia University Experiment Station. This support is gratefully acknowledged.

\section{REFERENCES}

[1] J. B. Brown, D. Y. Chung and P. W. Matthews, Heat pulses at low temperatures, Phys. Letters 21, 241-243 (1966)

[2] C. Cattaneo, Sulla conduzione del calore, Atti Sem. Mat. Fiz. Univ. Modena 3, 83-101 (1948)

[3] P. J. Chen, On the growth and decay of one-dimensional temperature rate waves, Arch. Rational Mech. Anal. 35, 1-15 (1969)

[4] P. J. Chen, On the growth and decay of temperature rate waves of arbitrary form, Z. Angew. Math. Phys. 20, 448-453 (1969)

[5] B. D. Coleman, Thermodynamics of materials with memory, Arch. Rational Mech. Anal. 17, 1-46 (1964)

[6] B. D. Coleman, On thermodynamics, strain impulses, and viscoelasticity, Arch. Rational Mech. Anal. 17, 230-254 (1964)

[7] B. D. Coleman and M. E Gurtin, Equipresence and constitutive equations for rigid heat conductors, Z. Angew. Math. Phys. 18, 199-208 (1967)

[8] B. D. Coleman and V. J. Mizel, Thermodynamics and departures from Fourier's law of heat conduction, Arch. Rational Mech. Anal. 13, 245-261 (1963) 
[9] B. D. Coleman and V. J. Mizel, Norms and semi-groups in the theory of fading memory, Arch. Rational Mech. Anal. 23, 87-123 (1966)

[10] B. D. Coleman and W. Noll, An approximation theorem for functionals, with applications in continuum mechanics, Arch. Rational. Mech. Anal. 6, 355-370 (1960)

[11] B. D. Coleman and W. Noll, Foundations of linear viscoelasticity, Rev. Modern Phys. 33, 239-249 (1961)

[12] B. D. Coleman and W. Noll, Simple fluids with fading memory, Internat. Sympos. Second-Order Effects in Elasticity, Plasticity and Fluid Dynamics (Haifa, 1962), Jerusalem Academic Press, Jerusalem; Pergamon Press, Oxford, 1964, pp. 530-552.

[13] W. A. Day and M. E. Gurtin, On the symmetry of the conductivity tensor and other restrictions in the nonlinear theory of heat conduction, Arch. Rational Mech. Anal. 33, 26-32 (1969)

[14] W. S. Edelstein and M. E. Gurtin, Uniqueness theorems in the linear dynamic theory of anisotropic viscoelastic solids, Arch. Rational Mech. Anal. 17, 47-60 (1964)

[15] M. E. Gurtin, On the thermodynamics of materials with memory, Arch. Rational Mech. Anal. 28, $40-50$ (1968)

[16] M. E. Gurtin and I. Herrera, On dissipation inequalities and linear viscoelasticity, Quart. Appl. Math. 23, 235-245 (1965)

[17] M. E. Gurtin and A. C. Pipkin, A general theory of heat conduction with finite wave speeds, Arch. Rational Mech. Anal. 31, 113-126 (1968)

[18] O. D. Kellogg, Foundations of potential theory, Springer, Berlin, 1939; reprint, Dover, New York, 1953.

[19] J. C. Maxwell, On the dynamical theory of gases, Philos. Trans. Roy. Soc. London 157, 49-88 (1867)

[20] V. J. Mizel and C.-C. Wang, A fading memory hypothesis which suffices for chain rules, Arch. Rational Mech. Anal. 23, 124-134 (1966)

[21] C. Truesdell and R. Toupin, The classical field theories, Handbuch der Physik, Band III/I, Springer Berlin, 1960, pp. 491-529 